\title{
Real-Time Aerodynamic Flow and Data Visualization in an Interactive Virtual Environment
}

\author{
Richard J. Schwartz ${ }^{1}$, Gary A. Fleming ${ }^{2}$ \\ ${ }^{1}$ Swales Aerospace, NASA Langley Research Center, Mail Stop 493, Hampton, VA 23681 \\ Phone: (757) 864 - 4597, Email: r.j.schwartz@larc.nasa.gov \\ ${ }^{2}$ NASA Langley Research Center, Mail Stop 493, Hampton, VA 23681 \\ Phone: (757) 864 - 6664, Email: Gary.A.Fleming@nasa.gov
}

\begin{abstract}
Significant advances have been made to non-intrusive flow field diagnostics in the past decade. Camera based techniques are now capable of determining physical qualities such as surface deformation, surface pressure and temperature, flow velocities, and molecular species concentration. In each case, extracting the pertinent information from the large volume of acquired data requires powerful and efficient data visualization tools. The additional requirement for real time visualization is fueled by an increased emphasis on minimizing test time in expensive facilities. This paper will address a capability titled LiveView3D, which is the first step in the development phase of an in depth, real time data visualization and analysis tool for use in aerospace testing facilities.
\end{abstract}

Keywords - Real time, data visualization, wind tunnel, virtual environment, optical metrology

\section{INTRODUCTION}

Thorough aerodynamic analysis of any proposed vehicle configuration requires a careful assessment and understanding of the off-body flow physics, on-body surface flow properties, and structural aeroelastic characteristics. Although computational fluid dynamics (CFD) and finiteelement modeling (FEM) codes can now provide predictions of these aerodynamic characteristics, a significant portion of vehicle aerodynamic assessment is still accomplished by wind tunnel testing. A number of different optical diagnostic techniques currently exist for measuring the flow and structural properties of wind tunnel models under test ${ }^{1}$. Extracting knowledge from these extensive data sets, often gigabytes per test condition, requires efficient and revealing data visualization methods. Most data produced by currently available optical instrumentation techniques are processed off-line, and full analysis may not occur until weeks or months after the test. However, the cost of wind tunnel operations and the complexity of interrelated aerodynamic phenomena being investigated are pushing the requirement of real-time data visualization and analysis.

Laser light sheet flow visualization is one of the simplest and most widely used optical diagnostic techniques for wind tunnel testing. This technique allows a researcher to qualitatively visualize off-body flow structure for identification of prominent flow features, such as the location of vortices, shear layers, shocks, etc. Laser light sheet flow visualization is accomplished by forming the output beam of a moderate-power continuous wave laser (typically an Argonion laser) into a thin, planar sheet of laser light. The sheet of laser light is then directed into the wind tunnel test section and aligned to illuminate the region-of-interest (Figure 1). Smoke or other particulate seeding is introduced into the flow upstream of the test section, which follows the flow streamlines as it convects downstream. The smoke particles ultimately pass through the light sheet and scatter the laser light, highlighting predominant flow features.

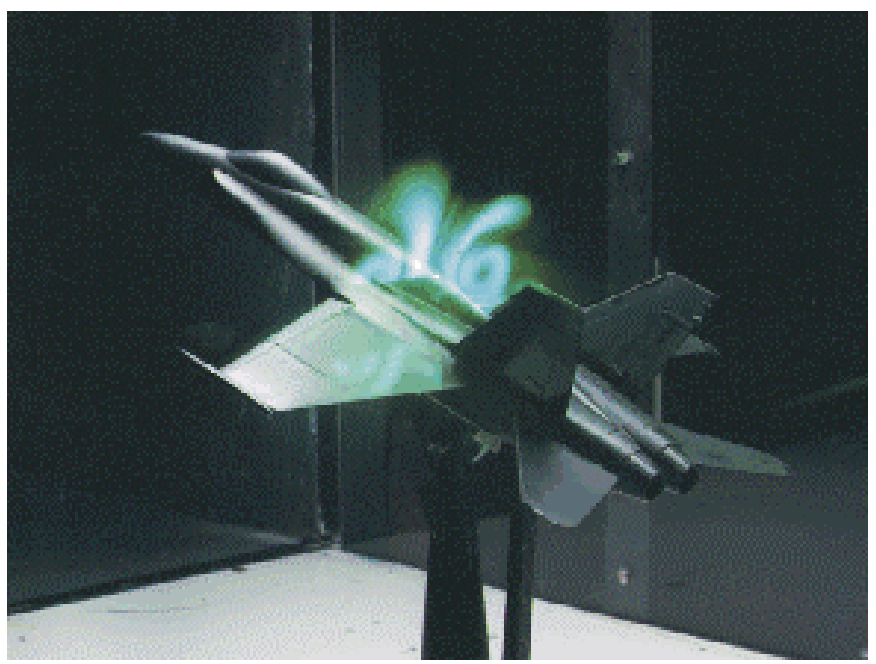

Figure 1. Typical application of laser light sheet flow visualization. Here the vortical flow structures above an F/A-18 aircraft are being investigated.

The flow visualization patterns are typically observed and recorded using a video camera and recording equipment. Although this technique is a very powerful tool for qualitative flow assessment, some flow features can be difficult to identify because of reduced imaging quality and the inability to change the camera view of the light sheet without adjusting the optical configuration. Additionally, it can be difficult to conceptually relate the locations of off-body flow structures relative to structural features on the test article (for example, visualization of vortex core that has propagated downstream relative to a leading-edge strake). Displaying the real-time laser light sheet flow visualization imagery in an interactive virtual environment offers a solution to several of these problems, and offers a way to enhance standard flow 
visualization techniques to provide greater knowledge to the researcher.

This paper addresses the LiveView $3 D$ enhanced flow visualization system recently developed and implemented at NASA Langley Research Center ${ }^{2}$. The LiveView3D system provides researchers the ability to view qualitative flow visualization imagery in real-time in an interactive, threedimensional virtual representation of the experimental test environment. LiveView3D allows researchers to interact with the virtual environment to view the flow visualization imagery from any angle, zoom in to investigate specific flow features, or apply transparency and false color effects to the real-time flow visualization imagery to accentuate details of interest. LiveView3D also increases empirical knowledge gained from conducting qualitative flow visualization experiments by spatially registering the flow visualization plane within the virtual test environment. This provides researchers with a better intuitive understanding of what flow features are occurring relative to the position and scale of the wind tunnel model. The LiveView3D system has been designed to accept video from industry-standard camera types, and can therefore be applied to different flow visualization techniques such as laser vapor screen, Schlieren, shadowgraph, or infrared thermography. The LiveView3D components, operation, and implementation are described in the forthcoming sections.

\section{TECHNICAL APPROACH}

The LiveView3D system is based on a PC-compatible computer using the Microsoft Windows ${ }^{\mathrm{TM}}$ operating system. The LiveView3D software consists of both commercially available off-the-shelf (COTS) components and customwritten code (Figure 2). The computer is also equipped with a COTS video frame grabber to capture video from the flow visualization camera.

\section{A. Hardware}

The process of capturing, enhancing, and displaying realtime video in an interactive virtual environment is computationally intensive. Therefore the computer used must have appreciable processing power. The current LiveView3D system is based on a dual $2.8-\mathrm{GHz}$ processor PC with $1 \mathrm{~Gb}$ random-access memory (RAM) and a workstation-class graphics card. The graphics card currently in use has its own graphics processing engine and custom application-specific integrated circuitry (ASICs) to accelerate graphics rendering operations. The graphics card also drives a dual monitor display, which is a feature the LiveView3D software has been written to take advantage of. The workstation-class graphics card is considered to be the key element for smooth graphics rendering and real-time interaction with the virtual environment. The video frame grabber used in the current LiveView3D system is a Matrox Millenium-II Multichannel frame grabber card that supports video capture from either one industry standard color NTSC video camera or up to four greyscale RS-170 video cameras. Other Matrox frame grabbers could be added or substituted to accommodate different camera interfaces (such as CameraLink, IEEE-1394, etc.). The Meteor-II Multichannel board contains no on-board image processing capability. While frame grabber cards from other vendors could have been used, the Matrox family of video cards was chosen because of our previous institutional knowledge of these cards and their supporting software libraries.

\section{B. Software}

The LiveView3D software consists of two programs running concurrently in a symbiotic fashion; the Controller Interface (CI) and the Visualization Interface (VI).

The CI program is a custom-written executable program written using Microsoft Visual Basic. The $\mathrm{CI}$ is designed to give the user an easy to manipulate graphical user interface (GUI) to control the functions of the frame grabber, aspects of the 3D display, and recording and playback of the flow visualization imagery. The control of the frame grabber and image processing tasks are done through the use of the Matrox ActiveMIL ActiveX controls, an in-depth library of functions for frame grabber hardware control and image processing.

The VI is based on 3D Studio Max by AutoDesk. This COTS application provides high-end visualization, photorealistic rendering, and three-dimensional computer object manipulation. Typically, this application is used for high-end animation, architectural and mechanical component visualization. 3D Studio Max has a fully developed programming language (MaxScript) that allows the user to create and manipulate geometry, bitmap mapping properties, visualization routines, and the user interface. External programs can communicate with the MaxScript language via the Microsoft ActiveX protocol. This is the mechanism that allows the CI to communicate with and control elements of the VI. For LiveView3D, additional custom MaxSript codes were developed to enable video to be streamed into the virtual environment.

\section{OPERATION}

The optimal use of the LiveView3D system requires the advance preparation of a realistic three-dimensional scale computer model, or geometry, of the experimental test facility. Although not essential, the inclusion of the test facility allows the user to gain insight into the global scope and potential impact any portion of the facility may have on the data set. The test facility can either be modeled directly from blueprints, dimensioned photographs, or physical measurements using 3D Studio Max, or imported into 3D Studio Max from pre-existing CAD files. Dynamic features of the experimental facility such as the motion of the model support system can also be incorporated into the geometry so that the motions of mechanically linked components 
realistically match between the virtual and actual environments.

The first essential step in operating the LiveView3D system is to import the test article geometry (i.e. wind tunnel model geometry) into 3D Studio Max. The test article geometry is typically the same three-dimensional assembledview CAD file used to fabricate the actual test article. If this file is not available, tools within 3D Studio Max can be used to generate a test article geometry of sufficient fidelity for qualitative flow visualization. The test article geometry is typically merged and scaled with the test facility model to create a complete and accurately scaled representation of the experimental environment.

The next step is to manipulate the virtual test article and/or test environment to replicate the different configurations that will be tested. In wind tunnel testing, this typically consists of changes in wind tunnel model pitch (angle of attack), roll, and yaw (sideslip), and any wind tunnel model translation within the test section. The fidelity of the virtual representations of the different test conditions depends on the ability to accurately align the virtual test article within the virtual environment. This can be facilitated by performing a relative alignment of key points on the virtual test article geometry to well known stationary reference points in the virtual experimental facility. Often physical measurements need to be made in the actual facility to determine the relative positions of these registration points. Once the virtual test configurations are finalized, they can be saved in individual geometry files. The virtual positions of the key registration points are also stored in a single geometry file. This way, different test article configurations previously stored to disk can simply be "snapped" into place when imported into the virtual test environment. Replicating the actual test configurations can be performed using controls provided within LiveView3D, or alternatively by using 3D Studio Max directly.

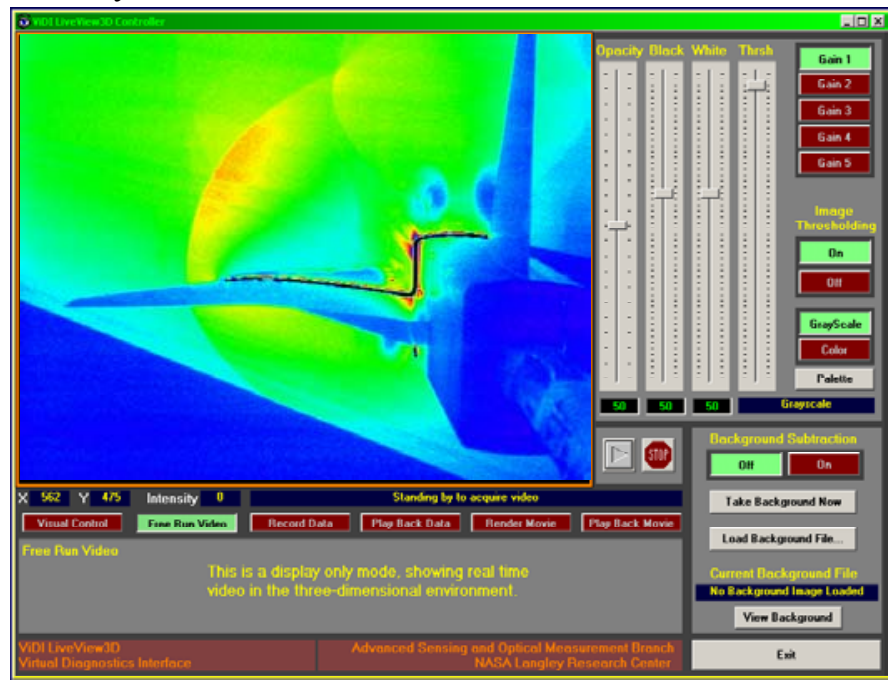

After the virtual test environment is created, a representation of the flow visualization plane is required. In the case of laser vapor screen flow visualization, this plane represents the laser light sheet. This plane is modeled as a thin box, which will ultimately have the video image texture mapped to it. The texture mapping is done using custom MaxScript codes that create a material that the incoming video can be texture mapped to. This material, when applied to the thin box, produces the effect of the real-time video being displayed in the virtual environment. The amount of self-illumination and opacity are set by this software, and can be changed in real time to enhance the visualization. Scaling the video to the correct size is accomplished by changing the size of the thin box. A known reference, such as a wingtip, can be used to match scale. Alternatively an object of known size (such as a tape measure) can be imaged with the flow visualization camera and the proper scale can be set through the use of a virtual ruler. The determination of new scaling factors only needs to be repeated if the spatial resolution of the flow visualization system changes. This might occur if different camera lenses are used to achieve different fieldsof-view, or if the camera- to light sheet- distance changes.

Experience has shown that background light and flare, or the strong reflections of the laser light off of the model surface, can degrade the quality of the light sheet visualization. In order to minimize these effects, LiveView3D has the ability to subtract background images from the data visualization images. This feature is implemented by acquiring and storing background images for each test condition planned. For laser vapor screen flow visualization, the background images are acquired with laser illumination and ambient lighting as they would be for the remainder of the test, but without seed material or smoke in the flow. If the wind tunnel model is expected to noticeably move or deform between its wind-off and wind-on conditions (model deflection is common in many tests), then it is

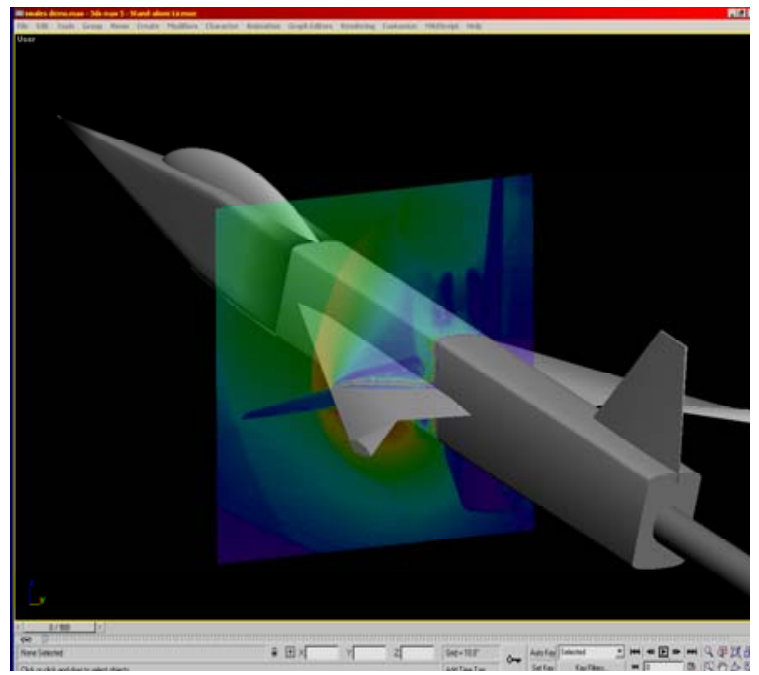

Figure 2. Cl user interface on left, and VI interface on right, as they appear during real time operation of LiveView3D. 
preferred to capture the background images while the tunnel is running at the test condition. Typically ten background images are acquired and averaged, and the average image is saved and subsequently used for background subtraction.

The flow visualization experiment can now be conducted. During these operations, LiveView3D is placed in "Free Run" mode. The video from the flow visualization camera is streamed through the LiveView3D system, and it appears in the virtual environment in real time. The user now has the freedom to optimize several parameters to enhance the visualization. Frame grabber controls such as gain, brightness, and contrast can be adjusted, as well as a low level threshold, which sets all video to a zero pixel value if it lies below a certain intensity value. Used in conjunction with the background subtraction, this significantly helps eliminate visually distracting low-level noise in the system. The user also has the choice of viewing the video stream in grayscale, or applying false color. Often during setup the most easily understandable color scheme is standard grayscale. However, to enhance detail and structure in the flow images, various false color schemes can be extremely beneficial. Each color palette is derived from a file containing values that connect pixel intensities to red, green and blue color components. Changing palettes can be done in real time by simply choosing a different palette file.

At any point while LiveView3D is operating, the user can manipulate the visualization in real time. So as the video data is streaming in, the user can view the virtual test configuration from any angle, and from any distance. The opacity of the flow visualization imagery or the test article can be changed, and specific elements of the virtual environment can be hidden or made visible.

Once the quality of the visualization is set as desired, the video stream may be saved to hard drive. The user must specify a file path and name, then a sequence of numerically numbered bitmap images are saved to hard drive while the flow visualization is occurring. This has only a slightly noticeable effect on the frame rate of the visualization. By saving the video data as independent bitmap image files, LiveView3D can then access the files after the test, substituting the live video with the stream of disk files. Thus, the same dataset can be played back as many times as desired, yet viewed under different conditions each time.

In addition to playing back the data in the interactive virtual environment, a movie file may be rendered. Using LiveView3D, The user may specify the view and the dataset to use, and have the computer create an animation. Since 3D Studio Max is designed as an animation program, the output will be a very high quality, photo-realistic representation of the visualization. It may take several seconds per frame for the animation to render, so this process is not real time. This animation file will be in a standard movie file format, enabling playback on almost any computer. Although this process is not executed in real-time, it has the advantage of rendering the entire virtual test environment and in-situ video stream to disk for later replay and distribution.

\section{IMPLEMENTATION}

LiveView3D has initially been deployed in the NASA Langley Research Center (LaRC) Unitary Plan Wind Tunnel (UPWT), and is slated for deployment in other NASA and United States Air Force facilities. As with all techniques, it has matured from its initial state when first demonstrated in used in a small research wind tunnel at NASA LaRC.

\section{A. Initial trial runs - The beta version}

The initial version of LiveView3D was very simple. A direct stream of grayscale video was captured and displayed in the virtual environment. The user interface consisted of an "on" and "off" button, and the only control the user had over the video display was the level of opacity the video would appear to have in the visualization.

First tested in a very small wind tunnel on a delta wing model, the video image of the flow visualization had excellent contrast and brightness. This produced distinct imagery in the virtual environment, and the encouragement to proceed with software development and move to larger facilities.

\section{B. First UPWT Run - Version 1.0}

The LaRC UPWT is a closed-loop, variable density, continuously running supersonic wind tunnel with two independent 4-x 4-x 7-foot test sections ${ }^{3}$. One test section operates at speeds from Mach 1.5 to 2.9, while the second test section operates at speeds form Mach 2.3 to 4.6. Laser vapor screen flow visualization is used often in the facility to investigate off-body flow features about the test article. Flow seeding is accomplished by injecting a small amount of liquid water into the tunnel while operating. The water evaporates in the low density flow and re-condenses when accelerated to supersonic speeds. This produces a uniform "fog" throughout the test section that is ideal for laser vapor screen flow visualization. Since laser vapor screen flow visualization is so often used in the facility, implementing LiveView3D in the UPWT was considered an excellent opportunity to demonstrate the benefits of the system.

Several features had to be added to the LiveView3D software before using it in an operational test facility. A user interface was designed to provide functionality while remaining easy to use. This included a window that showed the incoming video stream directly from the video camera, and an interactive slider to control the opacity of the video as it was projected into the virtual environment. The ability to save and replay the data in the virtual environment was also added to the software. And lastly, instead of using relying on the more complex standard interface in 3D Studio Max, a simple way to create and save animated renderings of the virtual test environment and in-situ video stream was developed.

The LiveView3D system was then taken to the UPWT and configured to accept flow visualization imagery from Test Section 1 - Mach 1.5 to 2.9. A generic supersonic aircraft 
configuration was used as the wind tunnel model (Figure 3). This model produced distinct shock waves and vortices at moderate angles of attack, providing ideal operational environment to gain experience with LiveView3D. The flow visualization camera used for this test was a small, "lipstick" sized analog CCD camera mounted in a housing on the model support system. The view was directly from the back of the model. This was an excellent vantage point, since there was virtually no perspective distortion, and very little optical blockage due to hardware obstructions in the video imagery.

As the test was being conducted, it became apparent that the LiveView3D system, while functionally properly, was not providing the type of dramatic visualization expected. Even with the highest laser power and the optimum seeding in the wind tunnel the video feed lacked brightness and contrast, so the resulting display of the grayscale imagery in the virtual environment suffered. Since the video camera had no further adjustments available for improving video quality, enhancements to the LiveView3D software were required to achieve the full potential of the system.

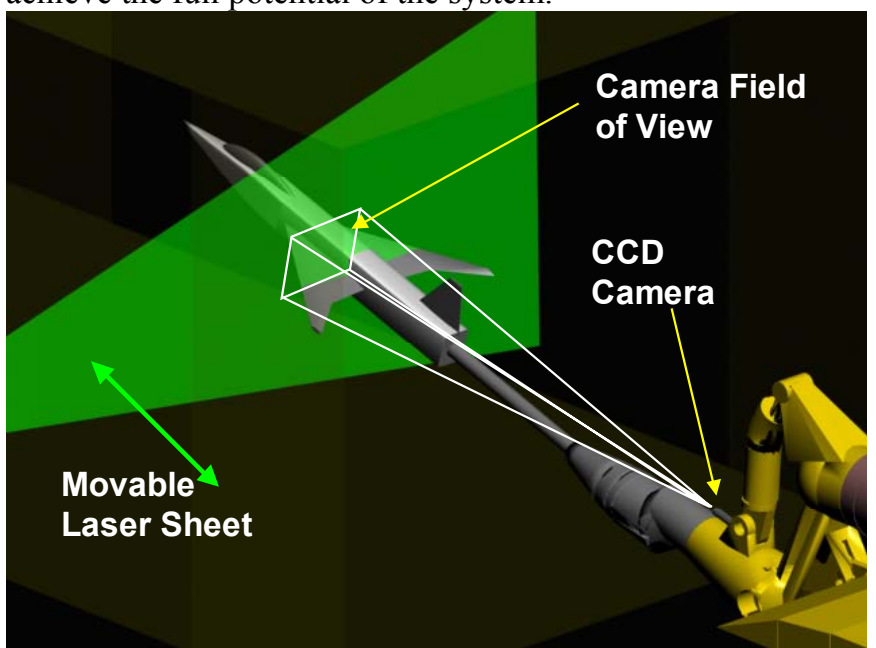

Figure 3. LiveView 3D experimental setup in the UPWT, using a generic supersonic fighter configuration.

\section{Second UPWT Run - Version 1.1}

Video amplification was required to further improve the quality and contrast of the imagery. The frame grabber supported five different gain levels, so the user was given controls to adjust the gain in real time. In addition, brightness and contrast adjustments were added. This boosted the input signal levels, yielding better results in the virtual environment. However, the added gain also amplified the noise level, thus keeping the signal-to-noise ratio constant. Two steps were taken to increase the signal-tonoise ratio. First, a real time background subtraction capability was added. This helped to reduce the glare off the model, lower the pixel levels outside of the region of interest, and removed fixed background features in the images. In addition, a user adjustable low-level threshold was employed. Any pixel values below the set value where set to zero, eliminating visually distracting low level noise.
The ability to apply false color enhancement to the incoming video stream was also added to the LiveView3D software. This allowed the user to choose from a variety of color palettes to accentuate different flow features. With this improvement, details in the video imagery became distinct and vibrant. Although the user now had a somewhat more complex interface to work with, the quality of the visualization was far superior to what was previously achieved.

The UPWT flow visualization experiment was repeated with the upgraded LiveView3D system. It took only a few moments to adjust the new controls to optimize the settings. The clarity of the flow visualization was dramatic. (Figure 4) While the video was steaming into the system, the visualization was manipulated in real time to view the data from all angles and levels of magnification, both with and without the wind tunnel facility visible. This elicited excited responses from the wind tunnel test engineers and technicians present. Several minutes of video was recorded at each test point in the matrix, allowing playback after the test.

Once the test was complete, data at the different test points were merged into one visualization (Figure 5). This was played back in an interactive mode, and was also rendered into a movie file. Before the system was finished documenting the flow field on this aircraft configuration, plans were made to add additional capabilities and to deploy the system for other wind tunnel tests.

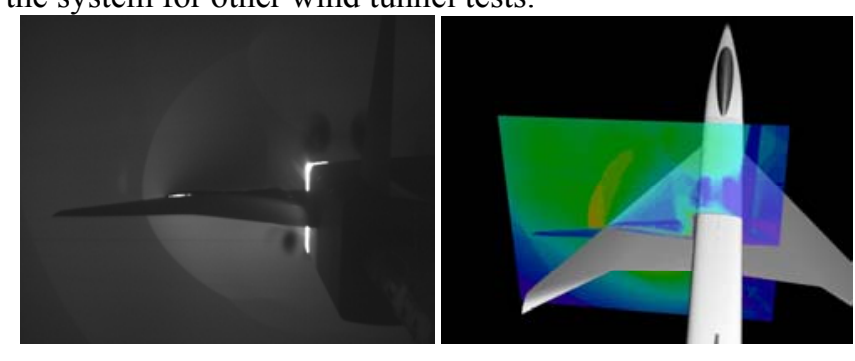

Figure 4. Comparison between original static flow visualization capability on TV monitor and interactive LiveView 3D output.

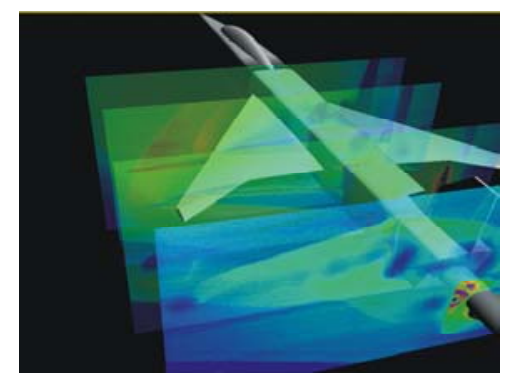

Figure 5. Merger of data from individual runs.

\section{FUTURE DEVELOPMENT}

There are several planned upgrades that are to be incorporated into future versions of LiveView3D. These include enhancements to the video capabilities as well as entirely new features to display a wide variety of data in both two and three dimensions in real time. 
In order to make the video portion of LiveView3 3 more versatile, a real-time image warping algorithm will be used to remove image distortions from camera perspective and lens distortions. This will require the addition of an extra calibration step during experimental setup, but will allow accurate spatial registration of the video data in the virtual environment and off-axis viewing of the flow visualization plane. During the experimental setup, a flat calibration plate with a regularly spaced array of dots at known spacing will be placed in the flow visualization plane. Images of this plate will be acquired with the flow visualization camera, and the imagery will be used to develop piecewise bilinear warping coefficients to computationally "straighten" the image. The warping algorithms can be executed in real-time, and no significant degradation is expected to the flow visualization frame rate displayed in the virtual environment.

A major addition to the software will be the ability conduct real-time communications with the wind tunnel Data Acquisition System (DAS). The major operational wind tunnel facilities at NASA Langley are all based on similar DAS architectures. Therefore the addition of this capability will be applicable to all major tunnels at NASA Langley, but will also be specific to the Langley DAS architecture. However, once the interface and data display mechanisms are established (as will be the case), transitioning the technology to a non-Langley facility will only require the development of new communications routines to communicate with a different tunnel DAS. The ability to communicate with the wind tunnel DAS allows scalar data from wind tunnel and model sensors to be displayed in real-time, along with the flow visualization video, in the virtual environment. This allows the unique visualization of parameters such as aerodynamic forces and moments (measured using straingauge balance system), model temperatures (measured using embedded thermocouples), or wing surface pressures (measured using arrays of surface pressure taps). This provides a intuitive mechanism to study aerodynamic causeand-effect relationships, whereby a researcher can view and correlate off-body aerodynamics with on-body properties. Additionally, interfacing with the tunnel DAS provides the ability to automatically change the virtual test configuration as the actual test configuration changes. For example, changes in actual wind tunnel model angle-of-attack can be communicated to the LiveView3D system, which can automatically change the virtual wind tunnel model angle-ofattack. Therefore an accurate representation of the true test environment can be maintained at all times.

The LiveView3D capability will also be extended to accommodate data produced by other global optical diagnostic techniques such as Projection Moiré Interferometry (structural deformation), Pressure Sensitive Paint (surface pressure distribution), Particle Image Velocimetry and Doppler Global Velocimetry (both off-body flow velocity). Each of these techniques supplies quantitative data as opposed to the qualitative data provided by the laser sheet visualization. But just like the laser light sheet visualization, each technique produces data in the form of an image that may be mapped into the virtual environment.

The information displayed in LiveView3D does not have to be generated experimentally. Mathematically derived data from Computational Fluid Dynamics (CFD) or finite element modeling (FEM) can be incorporated in the visualization. This will allow the experimental data and the computational data to be compared in real time as the experiment is being conducted.

Each new step in the development of the software will provide the research will an increasingly unprecedented amount of real time information in an intuitive, easy to understand format. The significantly enhanced situational awareness will provide a greater knowledge of the overall flow physics and instrumentation operation in real time, resulting in more informed and better quality decisions during the testing ${ }^{4,5}$.

\section{CONCLUSION}

LiveView3D has been developed by using of a variety of powerful tools for three-dimensional visualization, video capture and image processing. The system has been taken from a laboratory setting to a large test facility. Necessary changes were made to achieve success as the system matured, and further development will lead to more powerful and intuitive data visualization. Although development of LiveView3D has focused on the aerospace testing community, there are numerous applications in a wide variety of fields that can benefit from this technology.

\section{REFERENCES}

[1] Optical Metrology for Fluids, Combustion and Solids, Edited by Carolyn Mercer, Kluwer Academic Publishers, ISBN 1-40207407-7

[2] Schwartz, R.J., "ViDI: Virtual Diagnostics Interface Volume 1-The Future of Wind Tunnel Testing" Contractor Report NASA/CR-2003212667, December 2003

[3] Erickson, G.E., "Overview of Selected Measurement Techniques for External Aerodynamic Testing in a Supersonic Wind Tunnel Facility”, AIAA 2000-2396, AIAA Fluids 2000 Conference, 19-22 June, 2000, Denver CO.

[4] Jordan, J. D., Watkins, A. N., Fleming, G. A., Leighty, B. D., Schwartz, R. J., Ingram, J. L., Grinstead, K. D., Jr., and Oglesby, D. M., "Rapid Technology Assessment via Unified Deployment of Global Optical and Virtual Diagnostics", 20th International Congress on Instrumentation in Aerospace Simulation Facilities - ICIASF 03, Gottingen, Germany, August 25-29, 2003.

[5] Jordan, J. D., Watkins, A. N., Fleming, G. A., South, B. W., Leighty, B. D., Schwartz, R. J., Oglesby, D. M., Ingram, J. L., and Tominack, B. J., "Development of Rapid Technology Assessment Tools: A New Paradigm in Aerodynamic Design", ISA 49th International Instrumentation Symposium, Orlando, FL, May 4 - 8, 2003. 